\title{
Methotrexate-induced pulmonary toxicity
}

\author{
Baruch D Jakubovic BA ${ }^{1}$, Andrea Donovan MD FRCPC ${ }^{2}$, Peter M Webster MD FRCPC ${ }^{3}$, Neil H Shear MD FRCPC ${ }^{4}$
}

\begin{abstract}
BD Jakubovic, A Donovan, PM Webster, NH Shear. Methotrexateinduced pulmonary toxicity. Can Respir J 2013;20(3):153-155.
\end{abstract}

Methotrexate is a widely used medication with an array of recognized side effects. The present report describes a case of methotrexate-induced pneumonitis in a patient with psoriasis, and demonstrates the hallmark clinical and investigational findings that support this infrequently encountered diagnosis. The ensuing discussion reviews the pathogenesis, management and prevention of this adverse drug reaction.

Key Words: Methotrexate; Pneumonitis; Pulmonary toxicity

\section{Learning objectives}

- To recognize that methotrexate (MTX) causes pneumonitis that is associated with a constellation of nonspecific findings.

CanMEDS Competency: Medical expert

- To gain familiarity with the management and prevention of MTX-induced pneumonitis.

CanMEDS Competency: Medical expert \& communicator

Pretest

- What are the major clinical features supporting a diagnosis of MTX-induced pneumonitis?

- How can MTX-induced pneumonitis be managed and prevented?

\section{CASE PRESENTATION}

A 62-year-old Scottish-born woman presented to hospital experiencing progressive, nonproductive exertional cough accompanied by chest pain and dyspnea for the past four months. Other than recent nausea and emesis, she denied other constitutional or gastrointestinal symptoms.

The patient was a nonsmoker and denied exposure to asbestos. Her medical history was significant and included melanoma, type 2 diabetes mellitus, hypertension, hyperlipidemia, idiopathic angioedema, deep vein thrombosis, and a remote history of both uterine and breast cancer. In addition, she had a longstanding history of psoriasis not responsive to topical treatment. Oral MTX was, therefore, initiated 10 months before this presentation at a maximum of $20 \mathrm{mg}$ once per week combined with daily supplementation of $5 \mathrm{mg}$ of folic acid (except on the day she took MTX). Her other longstanding medications included ramipril, atenolol, atorvastatin, temazepam and acetaminophen/oxycodone.

On examination, she was afebrile. Her blood pressure was $153 / 87 \mathrm{mmHg}$, with a heart rate of 88 beats/min and a respiratory rate of 24 breaths/min. Initial oxygen saturation on room air was $88 \%$ but was increased to $93 \%$ on $50 \%$ oxygen by facemask at $8 \mathrm{~L} / \mathrm{min}$. There was adequate air entry bilaterally to the lungs, although fine bibasilar crackles were noted. The remainder of the physical examination was unremarkable other than her healing psoriaform rashes. Blood tests were within normal limits and an electrocardiogram was unremarkable. A chest $\mathrm{x}$-ray (CXR) revealed a diffuse interstitial pattern (Figure 1). Computed tomography (CT) of the chest revealed diffuse, ground-glass opacities (Figure 2). No effusions or lymphadenopathy were observed.

\section{Une toxicité pulmonaire induite par le méthotrexate}

Le méthotrexate est un médicament largement utilisé qui s'associe à une foule d'effets secondaires reconnus. Le présent rapport décrit un cas de pneumonite induite par le méthotrexate chez un patient ayant un psoriasis et présente les observations cliniques et les résultats d'examens caractéristiques en appui à ce diagnostic peu courant. L'exposé qui s'ensuit aborde la pathogenèse, la prise en charge et la prévention de cet effet indésirable du médicament.

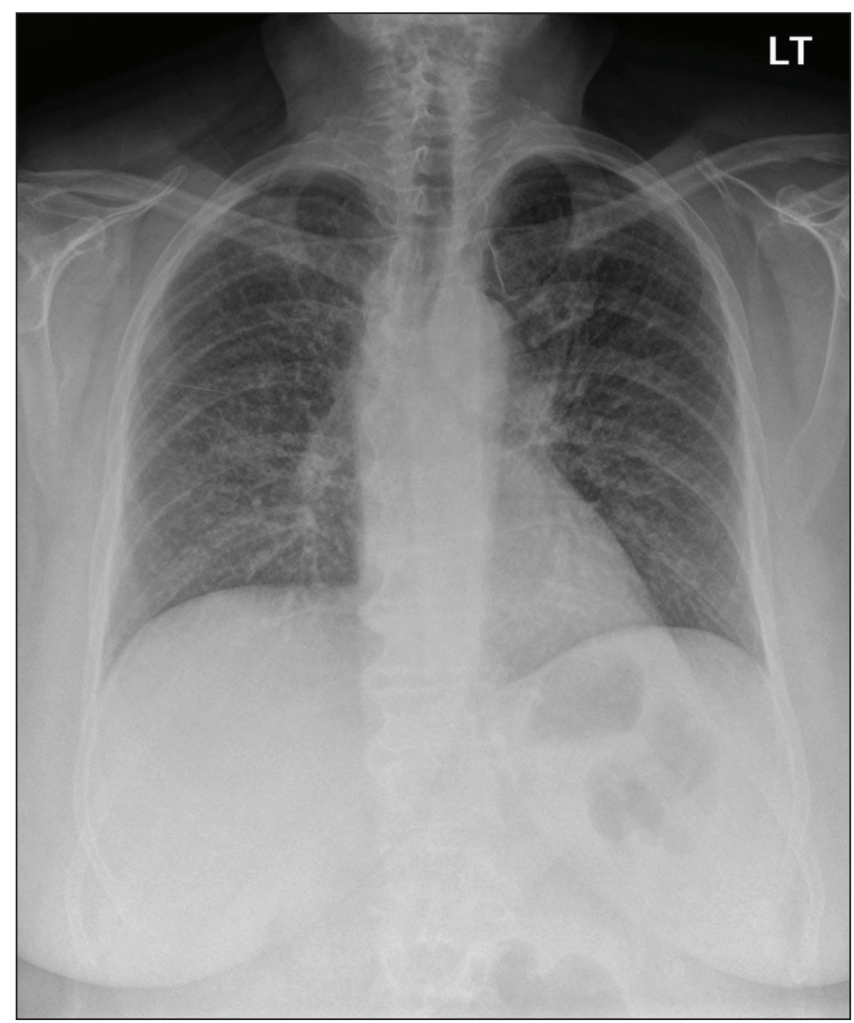

Figure 1) Initial frontal chest radiograph showing diffuse bilateral interstitial opacities

Cytology and culture of a bronchial washing detected no malignant cells or infection. Transbronchial biopsy identified nonspecific findings including chronic lymphocytic interstitial inflammation with reactive epithelial hyperplasia and focal aggregates of histiocytes within the alveoli. No granulomas were detected and microbiology stains were negative. Finally, trichrome staining demonstrated normal connective tissue content.

The patient's clinical presentation, in addition to subsequent investigational findings, supported a diagnosis of interstitial pneumonitis resulting from an idiosyncratic reaction to MTX. The patient was given oxygen by mask and levofloxacin initially for the possibility of

${ }^{1}$ Faculty of Medicine, University of Toronto; ${ }^{2}$ Department of Medical Imaging; ${ }^{3}$ Department of Medicine, Division of Respirology; ${ }^{4}$ Department of

Medicine, Divisions of Dermatology and Clinical Pharmacology, Sunnybrook Health Sciences Centre Eु University of Toronto, Toronto, Ontario

Correspondence: Dr Neil H Shear, Sunnybrook Health Sciences Centre, 2075 Bayview Avenue, M1-737, Toronto, Ontario M4N 3M5.

Telephone 416-951-2130,e-mail neil.shear@sunnybrook.ca 


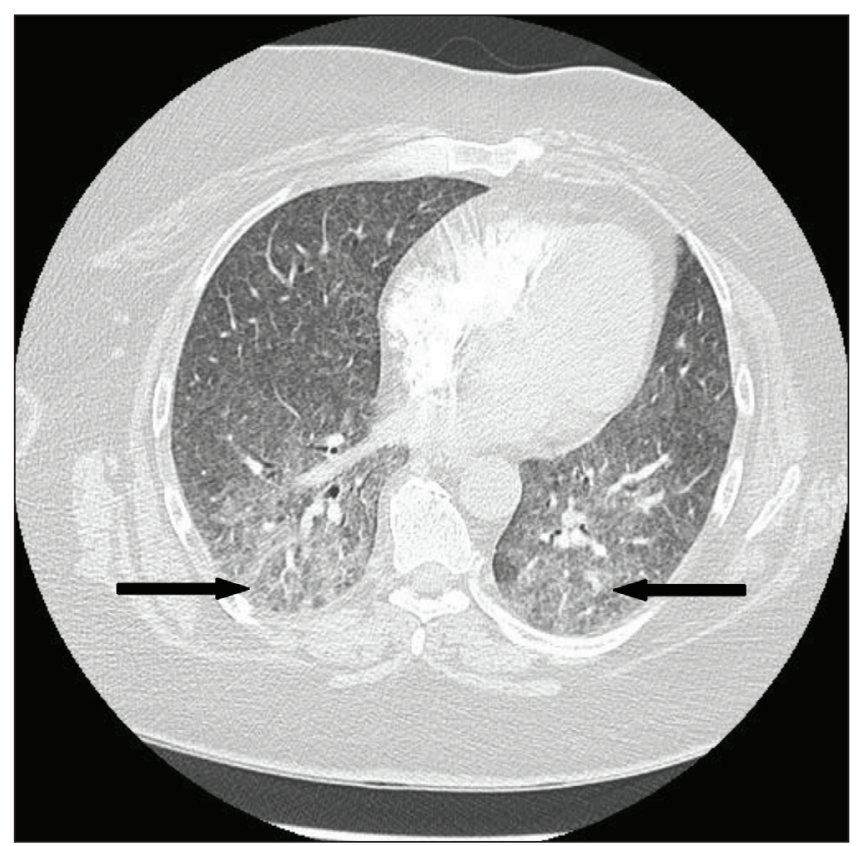

Figure 2) Axial computed tomography image through the mid lungs showing diffuse, bilateral ground-glass opacities (arrows). Of note, the imaging is devoid of evidence indicating additional or alternative pathology (ie, absent consolidation, atelectasis, effusions or lymphadenopathy

atypical pneumonia. MTX was discontinued, coinciding with improvement of her pulmonary disease, but resulted in worsening of her psoriasis. By the time of discharge (13 days following her presentation to hospital), her condition had improved both symptomatically (oxygen saturation on room air 92\%) and radiographically (Figure 3). Two weeks postdischarge, pulmonary function testing revealed a mild restrictive pattern, with total lung capacity and diffusion capacity of $78 \%$ and $68 \%$ of predicted, respectively. Subsequent to her discharge, she was treated with $40 \mathrm{mg}$ intramuscular triamcinolone acetonide to manage her flaring skin disease. These injections were continued every one to two months thereafter. While there is a clear link between autoimmune skin disorders and interstitial lung disease, psoriasis is one of the few disorders without this association. Thus, the resolution of her condition in response to MTX cessation confirmed that her respiratory complaints could be ascribed to an adverse drug reaction rather than underlying disease process.

\section{DISCUSSION}

As a folic acid analogue that antagonizes cellular proliferation, MTX can be used to manage a spectrum of inflammatory and neoplastic disorders. Approximately 1\% to $7 \%$ of patients receiving MTX treatment will develop pulmonary side effects $(1,2)$. Prompt recognition of interstitial pneumonitis is essential before it progresses to irremediable pulmonary fibrosis.

\section{Clinical features}

Patients characteristically present with a history of progressive respiratory complaints within one year of initiating MTX therapy (3). In psoriatic patients, MTX-induced pneumonitis has been documented after four months and up to 11 years following treatment initiation (4). Thus, recognition can be complicated by the late onset of symptoms.

Part of the difficulty in recognizing MTX lung toxicity also relates to the nonspecific symptoms voiced by patients, including progressive dry or productive cough and dyspnea, with or without fever $(1,5)$. Because no single test can confirm a diagnosis of MTX-induced pneumonitis, investigations serve to rule out other possible etiologies. CXRs will reveal a diffuse interstitial pattern not consistent with typical bacterial pneumonias - although Pneumocystis jirovecii

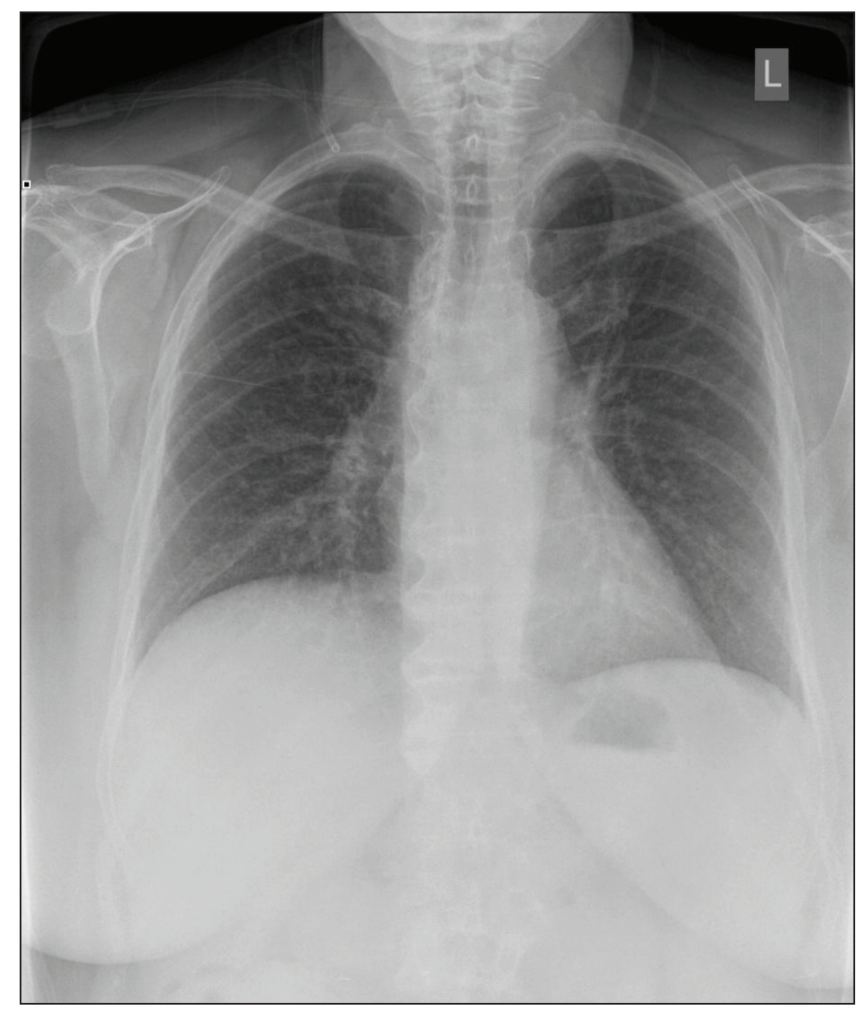

Figure 3) Repeat chest $x$-ray before discharge demonstrating radiographic improvement with increased lung aeration

and other atypical pneunomias may produce a similar radiographic pattern (1). CT will show characteristic ground-glass opacities with or without foci of consolidation (3). Evidence of restrictive lung disease will be apparent in pulmonary function tests. Bronchoalveolar lavage findings are nonspecific and include increases in both CD4 ${ }^{+}$ cell number and CD4/CD8 ratio $(3,6)$. Finally, lung biopsy (transbronchial and/or surgical) may also be performed and is generally indicated in instances of more severe or evolving respiratory disease in which cessation of MTX does not rapidly result in clinical improvement. Biopsy findings are also nonspecific, and may demonstrate evidence of acute pneumonitis with type II cell hyperplasia/ dysplasia and interstitial infiltration.

\section{Diagnostic criteria}

While different criteria for diagnosing MTX lung toxicity have been previously suggested $(3,5,7)$, they have not been clinically validated. MTX-induced pneumonitis is, thus, considered to be a diagnosis of exclusion (3).

\section{Pathophysiology}

Several mechanisms for the pathogenesis of MTX-induced pneumonitis have been surmised including hypersensitivity, direct drug toxicity to the lung tissue, or immunosuppression leading to repeated viral or other infections. Typical bronchoalveolar lavage and histological findings involving these patients support the concept that MTX-induced pneumonitis represents a hypersensitivity reaction (3). However, MTX also induces injury to alveolar epithelial walls, suggesting a direct drug toxicity route (8).

While several risk factors for MTX lung toxicity have been identified, they are limited to patients with rheumatoid arthritis. These include older age, previous disease-modifying antirheumatic drug treatment, hypoalbuminemia and diabetes mellitus, in addition to pleuropulmonary rheumatoid involvement $(1,9)$. Smoking has not been implicated in an increased incidence of MTX lung toxicity (3). 


\section{Management and prevention}

Cessation of MTX may itself be sufficient for symptom resolution and condition reversal (2). However, corticosteroid treatments have anecdotally been shown to be effective in accelerating symptom improvement - not unlike their utility in hypersensitivity pneumonitis (3). Patients presenting with severe hypoxia may require close monitoring and supplemental oxygen and/or assisted ventilation (2). Unlike the gastrointestinal and hematological complications than can result from MTX, the risk of pulmonary toxicity is not mitigated by folic acid supplementation. Furthermore, MTX lung toxicity is not typically associated with the gastrointestinal and hematological adverse effects that may ensue during treatment.

Outcomes for patients who experience MTX toxicity are usually good, with a low rate of progression to pulmonary fibrosis. However, a literature review involving 123 patients with suspected MTX lung toxicity reported a mortality rate of $13 \%$ (5). Nevertheless, despite the potential consequences of MTX lung toxicity, long-term monitoring with serial pulmonary function testing and CXR is not recommended (10). Thus, all patients prescribed MTX should be advised of the potential for lung toxicity and to report the development of respiratory symptoms to their physician (11). This will enable timely investigations to be initiated to search for the underlying etiology and, if no alternative can be identified, MTX treatment discontinued.

AUTHOR CONTRIBUTIONS: NS, PW and BJ were responsible for conception of the manuscript. All authors were involved in amalgamating the patient's clinical data. NS obtained formal consent from the patient who this report describes. BJ authored the initial draft of the manuscript. Revisions were performed by all authors who approved the final draft for submission.

\section{REFERENCES}

1. Kremer JM, Alarcon GS, Weinblatt ME, et al. Clinical, laboratory, radiographic, and histopathologic features of methotrexate-associated lung injury in patients with rheumatoid arthritis: A multicenter study with literature review. Arthritis Rheum 1997;40:1829-37.

2. Saravanan V, Kelly CA. Reducing the risk of methotrexate pneumonitis in rheumatoid arthritis. Rheumatology 2004;43:143-7.

3. Lateef O, Shakoor N, Balk RA. Methotrexate pulmonary toxicity. Expert Opin Drug Saf 2005;4:723-30.

4. McKenna KE, Burrows D. Pulmonary toxicity in a patient with psoriasis receiving methotrexate therapy. Clin Exp Dermatol 2000;25:24-7.

5. Imokawa S, Colby TV, Leslie KO, Helmers RA. Methotrexate pneumonitis: Review of the literature and histopathological findings in nine patients. Eur Respir J 2000;15:373-81.

6. Schnabel A, Richter C, Bauerfeind S, Gross WL. Bronchoalveolar lavage cell profile in methotrexate induced pneumonitis.

Thorax 1997;52:377-9.
ACKNOWLEDGEMENTS: The authors thank the patient who this report describes for consenting to have her case and medical imaging submitted for publication.

DICLOSURES: The authors have no financial disclosures or conflicts of interest to declare.

\section{Post-test}

- What are the major clinical features supporting a diagnosis of MTX-induced pneumonitis?

Both the clinical history and investigational findings are nonspecific. CXRs will demonstrate interstitial infiltrates that can be further visualized for the presence of fibrosis on CT. Bronchoalveolar lavage and/or lung biopsy can further help detect the presence of malignancy or infectious etiology. The absence of any findings suggestive of alterative pathology in conjunction with clinical improvement following MTX cessation confirms this diagnosis.

- How can MTX-induced pneumonitis be managed and prevented?

While folic acid supplementation is used to reduce MTX's other toxic effects, it does not mitigate the possibility of pneumonitis. In the acute setting, supportive care and oxygen supplementation in combination with discontinuing MTX are the mainstays of management, although corticosteroids may also be of benefit. Serial CXRs and pulmonary function testing are not recommended to monitor patients receiving MTX. The risk of pneumonitis should be communicated to patients so that they will seek appropriate medical care if concerning respiratory symptoms develop during the course of therapy.

7. Searles G, McKendry RJ. Methotrexate pneumonitis in rheumatoid arthritis: Potential risk factors. Four case reports and a review of the literature. J Rheumatol 1987;14:1164-71.

8. Ohbayashi M, Suzuki M, Yashiro Y, et al. Induction of pulmonary fibrosis by methotrexate treatment in mice lung in vivo and in vitro. J Toxicol Sci 2010;35:653-61.

9. Alarcon GS, Kremer JM, Macaluso M, et al. Risk factors for methotrexate-induced lung injury in patients with rheumatoid arthritis. A multicenter, case-control study. Methotrexate-Lung Study Group. Ann Int Med 1997;127:356-64.

10. Cottin V, Tebib J, Massonnet B, Souquet PJ, Bernard JP. Pulmonary function in patients receiving long-term low-dose methotrexate. Chest 1996;109:933-8.

11. Ameen M, Taylor DA, Williams IP, Wells AU, Barkert JN. Pneumonitis complicating methotrexate therapy for pustular psoriasis. J Eur Acad Dermatol Venereol 2001;15:247-9. 


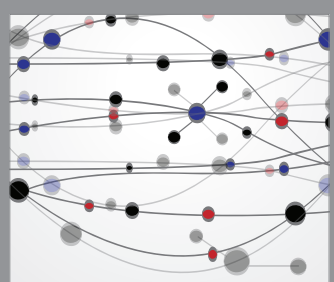

The Scientific World Journal
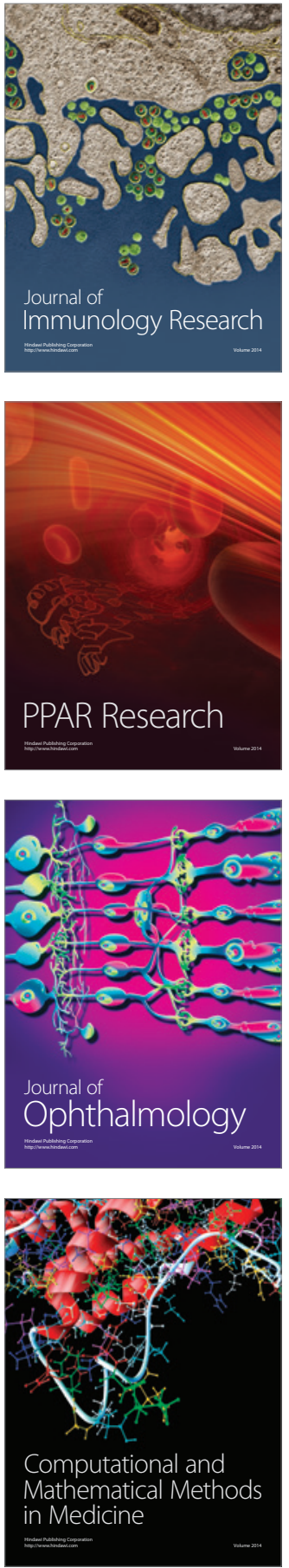

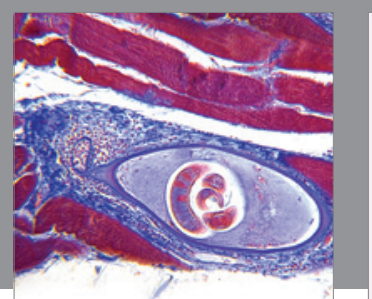

Gastroenterology Research and Practice

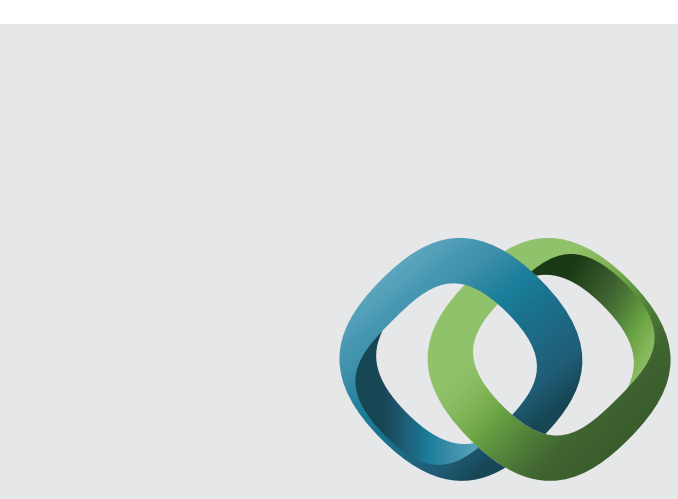

\section{Hindawi}

Submit your manuscripts at

http://www.hindawi.com
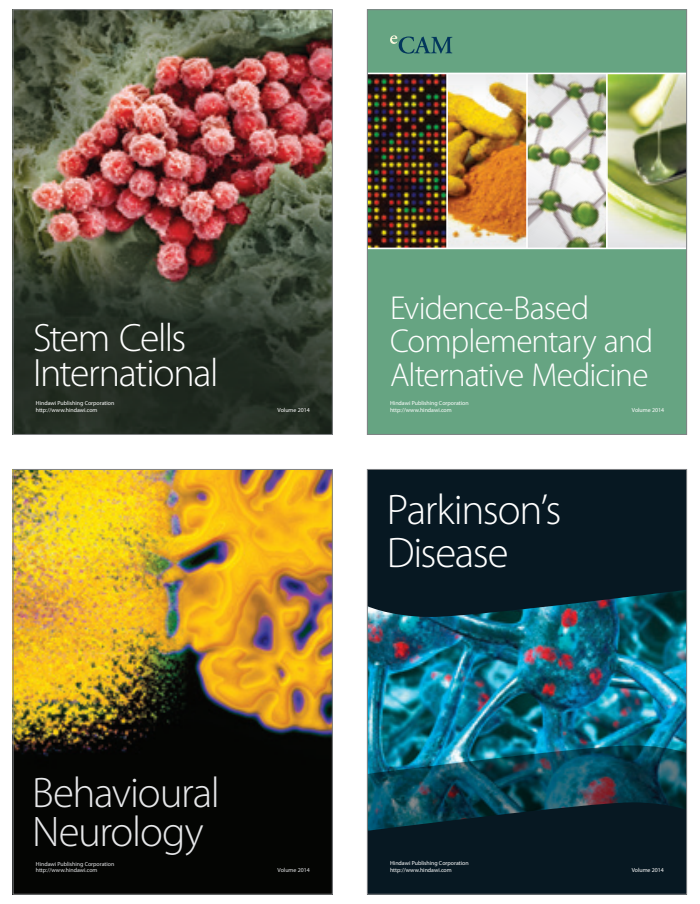
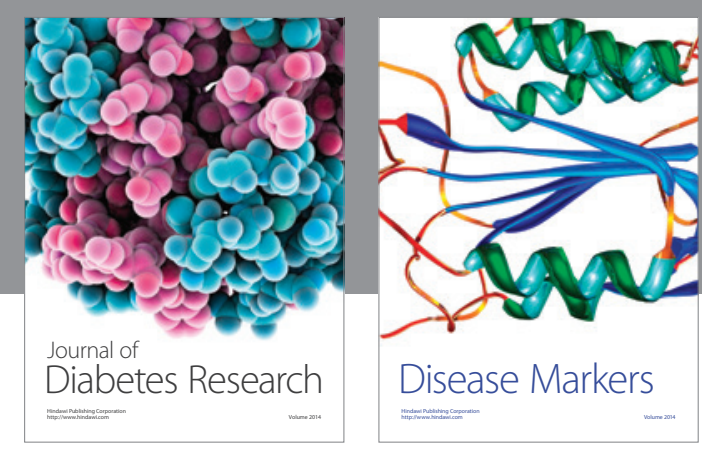

Disease Markers
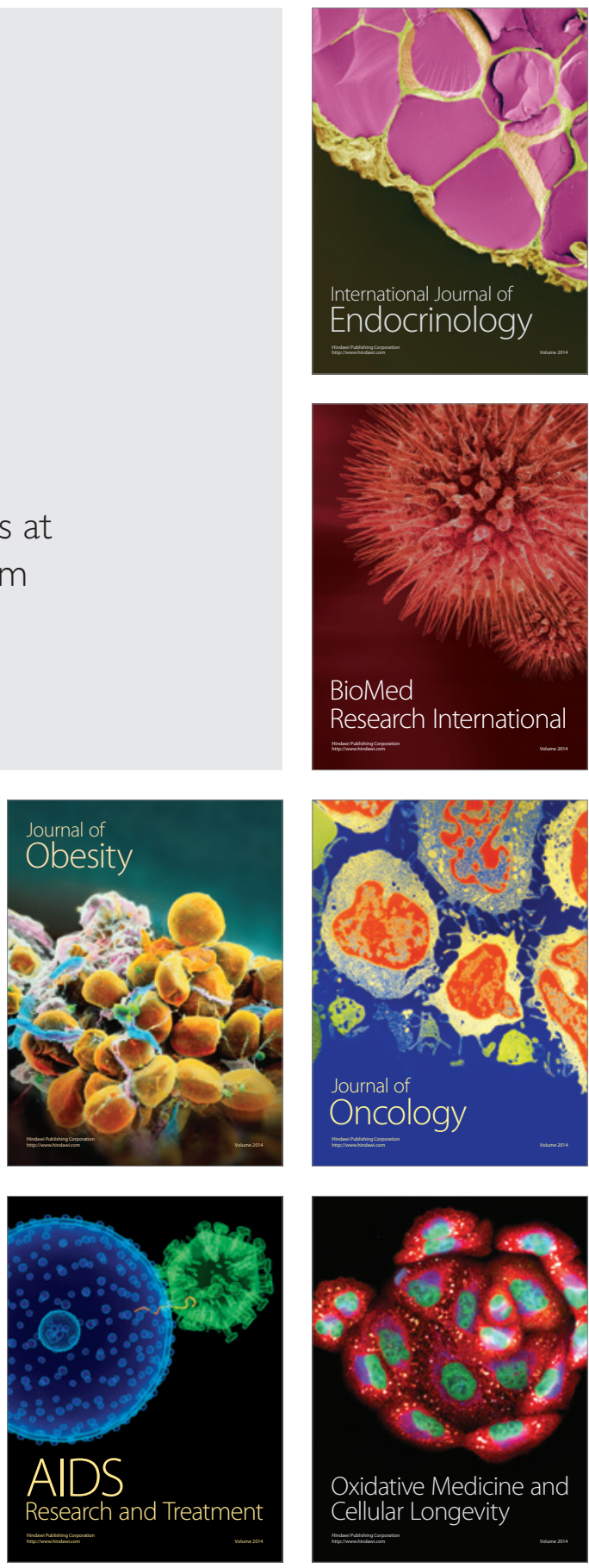